\title{
A Population-Based Time Determinant for Termination of Resuscitation
}

\author{
Michael G. Millin · Samuel M. Galvagno
}

Published online: 3 December 2010

(C) Société Internationale de Chirurgie 2010

To the Editor:

In a recent issue of World Journal of Surgery, Moriwaki et al. [1] present data regarding patients in cardiopulmonary arrest from blunt trauma. As discussed by the authors, resuscitation of a patient in cardiopulmonary arrest has significant cost $[2,3]$, and presents considerable risk to both prehospital providers and the public $[4,5]$. While not specifically discussed by the authors, survival without functional neurological outcome incurs additional costs, with prolonged admissions to acute care and rehabilitation facilities and a lifetime of required supportive care. Therefore, prehospital termination of resuscitation (TOR) protocols have the potential to contribute to public health through conservation of limited financial healthcare resources and prevention of further injuries by reducing the unnecessary use of warning lights and sirens.

Moriwaki et al. [1] recommend $20 \mathrm{~min}$ of resuscitation prior to TOR. While we appreciate that in this study of 477 patients in cardiopulmonary arrest from blunt trauma there were 13 survivors with a $2.7 \%$ survival rate, in analysis of the data presented in the authors' Table 1, it is evident that only 3 of these patients survived in a non-vegetative state, for a survival rate with good neurological outcome of $0.6 \%$. Additionally, and perhaps more importantly, from

\footnotetext{
M. G. Millin

Department of Emergency Medicine, Johns Hopkins Hospital, 600 N. Wolfe Street, Baltimore, MD 21287, USA

\section{S. M. Galvagno $(\bowtie)$}

Division of Adult Critical Care Medicine, Johns Hopkins Hospital, 600 N. Wolfe Street, Baltimore, MD 21287, USA

e-mail: sgalvag1@jhmi.edu
}

the authors' Fig. 8, it is clear that a cluster of 12 survivors achieved return of spontaneous circulation (ROSC) in under $10 \mathrm{~min}$, and a single isolated survivor achieved ROSC after $17 \mathrm{~min}$. No information is provided for this outlier. The initial rhythm, vital signs, and mechanism of injury are not described. Furthermore, it is unclear if this outlier was one of the three patients listed in the authors' Table 1 with a good neurological outcome.

In the development of a healthcare system, it is crucial to make decisions based on population-based data and not on individual outcomes. Moriwaki et al. [1] recommend TOR after $20 \mathrm{~min}$ of resuscitation based on the survival of one patient who had $17 \mathrm{~min}$ of resuscitation before achieving ROSC. However, their data plainly demonstrate that $10 \mathrm{~min}$ of resuscitation will capture the population of patients in cardiopulmonary arrest from blunt trauma.

Therefore, in analysis of the Moriwaki et al. [1] data, we recommend a population-based resuscitation time of 10 min prior to TOR.

\section{References}

1. Moriwaki Y, Sugiyama M, Yamamoto T et al (2010) Outcomes from prehospital cardiac arrest in blunt trauma patients. World $\mathrm{J}$ Surg 2010. 19 Oct [Epub ahead of print]

2. Stockinger ZT, McSwain NE (2004) Additional information in support of withholding or terminating cardiopulmonary resuscitation for trauma patients in the field. J Am Coll Surg 198:227-231

3. Rosemurgy AS, Norris PA, Olson SM et al (1993) Prehospital traumatic cardiac arrest: the cost of futility. J Trauma 35:468-474

4. Eckstein M (2001) Termination of resuscitative efforts: medical futility for the trauma patient. Curr Opin Crit Care 7:450-454

5. Centers for Disease Control and Prevention (CDC) (2003) Ambulance crash-related injuries among Emergency Medical Services workers-United States, 1991-2002. Morbid Mortal Week Rep (MMWR) 52:154-156 